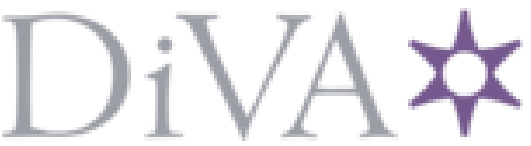

http://www.diva-portal.org

Preprint

This is the submitted version of a paper presented at The Proceedings of the 11th International Conference on Social Robotics (ICSR 2019).

Citation for the original published paper:

Technology, K R. (2019)

Knock on Wood: The Effects of Material Choiceon the Perception of Social Robots In: Lecture Notes in Artificial Intelligence series (LNAI)

N.B. When citing this work, cite the original published paper.

Permanent link to this version:

http://urn.kb.se/resolve?urn=urn:nbn:se:kth:diva-263923 


\title{
Knock on Wood: The Effects of Material Choice on the Perception of Social Robots
}

\author{
Sanne van Waveren, Linnéa Björklund, Elizabeth J. Carter, and Iolanda Leite \\ KTH Royal Institute of Technology, Stockholm, Sweden $\{$ sannevw, iolanda\}@kth.se
}

\begin{abstract}
Many people who interact with robots in the near future will not have prior experience, and they are likely to intuitively form their first impressions of the robot based on its appearance. This paper explores the effects of component material on people's perception of the robots in terms of social attributes and willingness to interact. Participants watched videos of three robots with different outer materials: wood, synthetic fur, and plastic. The results showed that people rated the perceived warmth of a plastic robot lower than a wooden or furry robot. Ratings of perceived competence and discomfort did not differ between the three robots.
\end{abstract}

Keywords: Social Robotics · Robot Material Design · Social Perception

\section{Introduction}

In science fiction, robots are often depicted as human-like or as metallic, machinelike creatures. Now, social robots are going beyond their roles in science fiction and taking a place in our daily lives. Currently, these commercially available robots for personal use do not look like realistic humans, and their component materials are often metal or plastic. However, it is possible to incorporate other materials into robot design, including wood, fabric, and fur, that could make robots more pleasing and comfortable to use in different contexts.

As many people interacting with robots will not have prior experience, they are likely to intuitively form their impressions of a robot based on its outer appearance during the first milliseconds of interaction, as is typical among humans [31]. In fact, people ascribe social attributes, such as warmth and competence, to social robots based on their physical characteristics [21].

While studies have been done on robot embodiment (e.g., physical vs. virtual $[11,15,30])$, robot body shape (e.g., size [19], waist-to-hip ratio and shoulder width [1]) and facial features (e.g., eyebrows, eyes [6,14, 26]), less attention has been given to component materials. Some works have investigated how robot skin can convey emotions through skin textures [13] and skin temperature [24]. One research team developed a robot with customizable skin [29]. However, the question of how the choice of component material affects people's perception of robots remains open.

Human-robot interaction (HRI) may benefit if people's perceptions of a robot can be shaped through choice of component material. In this work, we examine 
if the choice of outer material affects people's perception of a social robot; we study the effects of using wood, plastic, and synthetic fur on people's impressions of warmth, competence, and discomfort. We expect that a robot made of plastic (an inorganic material) will be rated less positively than a wooden or a furry robot. We expect fur to score similarly to wood, as both materials resemble organic materials. Specifically, we hypothesize that:

- H1: A robot constructed of wood will be rated as more positive than a robot constructed of plastic.

- H2: A robot constructed of fur will be rated as more positive than a robot constructed of plastic.

- H3: A robot constructed of wood will be rated equally positive as a robot constructed of fur.

\section{Related Work}

The design of a robot should match its intended function [6,17]; prior work has focused on different aspects of robot appearance, such as the body's shape and the design of the face. Social robots that are intended for human interaction should have a degree of humanness that evokes positive reactions from the people with whom they interact, without being too similar to humans, as this may elicit feelings of uncanniness [22].

To date, many works have investigated what level of the human-likeness is appropriate to increase the effectiveness of HRIs [7,9]. An fMRI study by Krach et al. [17] showed that a robot's degree of human-likeness influences people's cortical activity in regions associated with Theory of Mind [27]; perceived humanlikeness was positively correlated with the tendency to attribute mental states to the robot. However, Phillips et al. [25] argued that there is little systematic understanding of what constitutes a robot's human-likeness and identified four different dimensions (body-manipulators, facial features, mechanical locomotion, and surface look) that contribute to the perceived human-likeness of a robot. We discuss prior work on the facial features and surface appearance of social robots.

The face plays a crucial role in human interactions: it conveys information about what a person is thinking or feeling, their age and physical condition, and sometimes even their personalities. Early work focused on the mechanical design of expressive humanoid robot faces. For example, Hegel et al. [10] designed the social robot 'Flobi', which appears cartoon-like and can express both primary emotions (e.g., happy, sad) and even some secondary emotions with moderate success (e.g., shame). The design used baby-face cues (e.g., relatively large eyes, small chin, small lips and nose, and round head shape) to make the robot appear warmer, more naive, less dominant and less competent. DiSalvo et al. [6] conducted a study on the design and perception of a humanoid robot head and found that a humanoid robot head and face with a small forehead, small chin, and a head that is wider than that it is long, create a positive experience in HRI.

Luria et al. [20] studied the effects of a social robot's eye designs on how it was perceived. The authors created sixteen eye options for paper prototypes of 
a tabletop robot, ranging from lifelike eyes to abstract eyes, and no eyes as a control. The results showed that robots with more lifelike eyes were perceived as more personable and as a better fit for entertainment roles and domestic environments relative to robots with more abstract eyes or no eyes.

While robots typically have mechanical faces, rendering robot faces on a screen has endowed robots with more facial expressivity. Carpinella et al. [4] manipulated the masculinity vs. femininity and machine-likness vs. human-likeness of rendered robot faces and found that robots with a higher degree of humanlikeness and femininity were perceived as warmer, and machine-like or masculine robots were seen as more discomforting. Similarly, Kalegina et al. [14] studied people's preferences in realism and detail of rendered robot faces; the authors manipulated one face feature at a time and found that less realistic and less detailed robots are preferred in domestic settings and highly detailed, whereas not too realistic, robots are preferred for service tasks. Lehmann et al. [18] used a user-centered design approach to design the face display of a humanoid robot companion. An online survey showed that people rejected abstract designs for the robot face, and preferred an iconic face without too much details. In summary, the design of a robot face needs to consider the social role and function of the robot, as well as its human-likeness, gender facets, and realism.

Prior work focused mostly on the body shape and facial features of robots, and an open question is what material should be used for the surface of robots' bodies. Some research has looked into how robot skin can convey emotions using skin textures and form $[2,13]$ and skin temperature [24]. One work addressed the need for customizable robots with SPRITE, a socially assistive robot with a modifiable skin to tailor the robot to a target population or specific domain [29]. The authors stated that robot's small size and colorful outer appearance made people perceive it as non-threatening.

This work draws inspiration from other domains, such as industrial design, that have studied material choice in the process of product design. One study examined the link between the outer material of a bowl and what emotions it evoked within the participants [5]. It was found that wood scored high on all positive attributes measured, including joy and satisfaction, and low on negative attributes, such as disgust and fear. Contrarily, plastic generally scored high on negative and low on positive attributes. In this work, we investigate to what extent these effects translate to robots. Our work extends existing literature by investigating how surface material of a robot affects people's perception of its social attributes.

\section{Robot Design}

The robots were constructed from three different materials: birch plywood, birch plywood covered in synthetic fur, and acrylic plastic; see Figure 1. Besides that prior interior design research showed that wood scored higher on eliciting positive emotions and lower on negative emotions than plastic [5], these materials are easy to work with, being both cheap and workable with a laser cutter. The robots 
possessed three degrees of freedom with MG90s micro servos in the shoulder joints and the neck, using Arduino Uno for motion control.

\section{Method}

\subsection{Participants and Design}

120 participants were recruited from Amazon's Mechanical Turk (MTurk), they were compensated for their participation. As control questions, participants were asked to report the color of the square shown at the end of each video ("What color was the square at the end of the video?"), and we added the following item to the questionnaire: "To make sure you are still paying attention, we have added this question. Please choose 9 'definitely associated' for the next item 'check question'." Three workers were excluded from the analysis because they failed to answer two or more of the control questions correctly, resulting in a total of 117 participants (43 female and 74 male). Participants' mean age was $32.82(\mathrm{SD}=8.63)$, and they described themselves as White/Caucasian $(53.8 \%)$, Black or African American (5.1\%), Asian or Pacific Islander (26.5\%), Hispanic, Latino, or Spanish origin (7.7\%), Native American, American Indian, or Alaska Native $(2.6 \%)$, Native Hawaiian $(0.9 \%)$, mixed $(0.9 \%)$, unknown $(2.7 \%)$. A total of $56(47.9 \%)$ participants reported to have completed a bachelor's degree, 23 $(19.7 \%)$ participants a master's degree, $16(13.7 \%)$ participants some college credit but no degree, $14(12 \%)$ participants had a high school graduate, diploma or equivalent, four $(3.4 \%)$ completed trade/technical/vocational training, three (2.6\%) participants completed an associate degree, and one $(0.9 \%)$ reported to have completed a doctorate degree. Overall, participants reported that they were somewhat familiar with robots, with a mean of $2.4(\mathrm{SD}=1.1)$ on a scale from zero (not at all) to four (extremely). Specifically, 25 (21.4\%) participants reported to work with robots, $27(23.1 \%)$ participants study or have studied robots, nine $(7.7 \%)$ reported to own a robot, $73(62.4 \%)$ participants said to watch or read media that includes robots, and one participant reported to have no experience with robots. We manipulated within-subjects the independent variable of robot

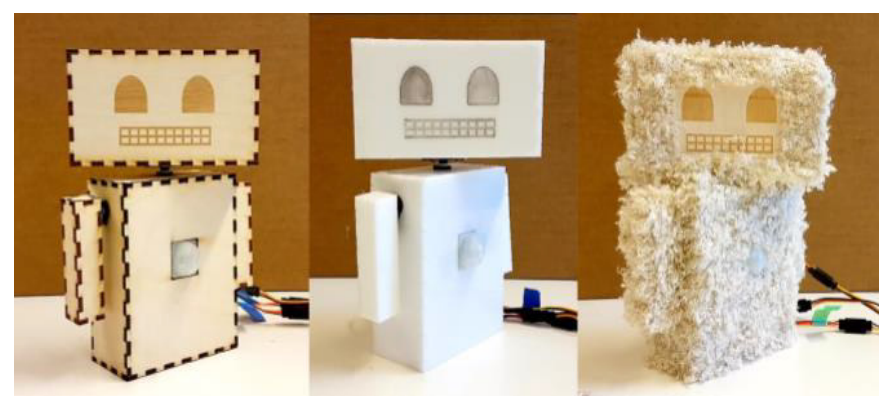

Fig. 1. The three versions of the robot: wood, plastic, and fur. 
material (wood vs. fur vs. plastic). To account for order effects, the order in which the robots were presented was counterbalanced.

\subsection{Materials and Procedure}

Participants viewed a video of each robot in which the robot was angled 45 degrees away from the viewer, then turned its head 45 degrees to face the viewer, raised the arm closest to the viewer and waved up and down three times ${ }^{1}$. After each video, participants were asked to rate the robot they just watched on warmth, competence, and discomfort dimensions on a scale from 1 (definitely not associated) to 9 (definitely associated) adapted from Carpinella et al. [4]. We included the following items as they were most relevant to the current study: reliable, competent, knowledgeable, and capable (resulting in four items on the competence dimension); organic, sociable, emotional, happy, and feeling (resulting in five items on the warmth dimension); and awkward, scary, strange, awful, and aggressive (resulting in five items on the discomfort dimension). All three dimensions for all three robots were found to have adequate internal consistency, with Cronbach's $\alpha$ levels all above 0.9. Therefore, we averaged the individual items of each dimension to obtain the final score for that dimension. Additionally, we asked how much they liked the robot $(1=$ dislike, $7=$ like $)$ and their willingness to interact/spend time with the robot $(1=$ not at all willing, $7=$ very willing). Finally, participants were asked to give each robot a name, to report which robot they preferred and why ("Please explain in a minimum of 20 words why you prefer this robot. This helps us understand your responses better."), and to provide demographic information.

\section{Results}

We analyzed the ratings on perceived warmth, competence, discomfort, likability, people's willingness to interact with each of the three robots, and which robot the participants preferred. To gain additional insight into why people preferred a specific robot, we performed qualitative analysis on the free-text answers.

\subsection{Social Attribute Judgments}

A repeated-measures ANOVA with skin type (wood vs. fur vs. plastic) as the within-subjects factor showed that warmth differed statistically significantly across materials $\left(F(2,232)=11.15, p<0.0005, \eta^{2}=0.09\right)$ (See Fig. 2). Post hoc tests using the Bonferroni correction revealed that the plastic robot scored statistically significantly lower $(\mathrm{M}=4.72, \mathrm{SD}=2.35)$ than the wooden $(\mathrm{M}=5.10$, $\mathrm{SD}=2.25, p<0.005)$ and the furry robot $(\mathrm{M}=5.19, \mathrm{SD}=2.15, \mathrm{p}<0.0005)$. Wood and fur did not differ significantly from each other $(p=1.0)$. In line with the low ratings of warmth for the plastic robot, examples of names that the participants gave the plastic robot include "Snowman robot", "ice cold robot", 


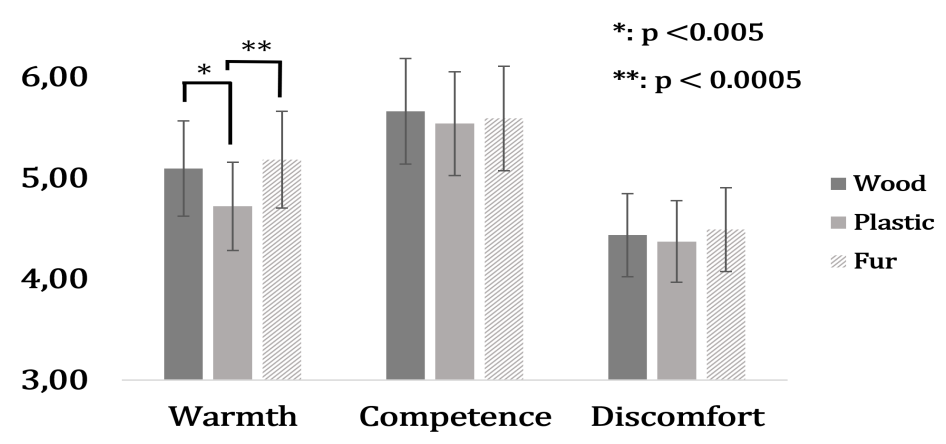

Fig. 2. Mean warmth, competence, and discomfort ratings. Error bars indicate standard errors.

and "pale robot". Both ratings for competence and discomfort did not differ statistically significantly. A repeated-measures ANOVA for skin type showed no significant difference in perceived competence $(F(2,232)=1.28, p=0.28$; wood: $\mathrm{M}=5.57, \mathrm{SD}=1.94$, fur: $\mathrm{M}=5.45, \mathrm{SD}=2.09$ and plastic: $\mathrm{M}=5.44$, $\mathrm{SD}=2.10)$. A repeated-measures ANOVA for skin type showed no significant difference in discomfort $(F(2,232)=0.77, p=0.46$; wood $\mathrm{M}=4.55, \mathrm{SD}=$ 2.41 , fur: $\mathrm{M}=4.58, \mathrm{SD}=2.44$ and plastic: $\mathrm{M}=4.44, \mathrm{SD}=2.46$ ).

\subsection{Likability}

The participants rated the three robots moderately on likability. The wooden $(\mathrm{M}=5.14, \mathrm{SD}=1.6, \mathrm{Mdn}=5.0)$ and furry $(\mathrm{M}=5.21, \mathrm{SD}=1.66, \mathrm{Mdn}=6.0)$ robots were rated slightly higher than the plastic robot $(\mathrm{M}=4.97, \mathrm{SD}=1.6$, Mdn $=5.0$ ), but a repeated-measures ANOVA showed that the scores did not differ significantly $(p=0.096)$.

\subsection{Willingness to Interact}

The participants rated their willingness to interact with the thee robots as moderately high (furry robot $\mathrm{M}=5.26, \mathrm{SD}=1.54, \mathrm{Mdn}=6.0$; wooden robot $\mathrm{M}=$ $5.16, \mathrm{SD}=1.60, \mathrm{Mdn}=6.0$; plastic robot $\mathrm{M}=5.09, \mathrm{SD}=1.63, \mathrm{Mdn}=5.0$ ). A repeated-measures ANOVA found no statistically significant difference in the mean scores of the three robots $(p=0.37)$.

\subsection{Robot Preference}

$45(38.5 \%)$ participants reported preferring the plastic robot, $40(34.2 \%)$ preferred the furry robot, and $32(27.4 \%)$ preferred the wooden robot; statistically, the three robots were not unequally preferred; $\chi^{2}(2)=2.205, p=0.33$. This suggests that despite the difference in ratings on the warmth dimension, participants

\footnotetext{
${ }^{1}$ Videos are available at bit.ly/2IsnMSW, bit.ly/2Iph28i, bit.ly/2L1vBAz
} 
prefer the three types of robots equally. To gain insight into participants' robot preferences, two independent coders coded the open-ended question in which participants were asked to explain their choice and we report the main findings. 101 out of 117 answers were coded with substantial inter-rater agreement (fuzzy $\kappa[16]=0.76)$, the other 16 answers were either unrelated or incomprehensible.

People's reason to prefer the furry and wooden robots seems to often relate to their material, which reminds people of pets or wooden objects they have seen before (kid's toy or cartoon), and their social character traits, such as kindness and friendliness, e.g., "I prefer this robot because it us furry, which makes it look more animal like and friendly. It's almost like a pet. I think it's cute." (Fur robot) and "Something about the design makes it feel more like a kids toy or a cartoon. The dashes that run around the edges kind of remind me of animated characters. (...) it reminds me of Awesom-O (from the TV show South Park)." (Wooden robot). The people who preferred the plastic robot seem to comment positively on its appearance and usefulness, e.g., "I like this white color robot and $i$ think this one is very knowledgeable, responsive, capable robot [more] than the other robots".

\section{Discussion and Conclusion}

Robot design is a crucial aspect of HRI research and in this study, we shed some light on the role of robot component material and pave the way for further research on the effects of robot materials on people's perceptions of social robots. We hypothesized that a robot constructed of wood would be rated more positively than a robot constructed of plastic $(H 1)$, that a robot constructed of fur would be rated more positively than a robot constructed of plastic (H2), and that a robot constructed of wood would be rated equally positively as a robot made of fur (H3).

The results showed that people rated the plastic robot significantly lower on perceived warmth as compared to the wooden and furry robots. The warmth ratings for the wooden and furry robots did not differ significantly, which confirms all three hypotheses for the warmth dimension. Interestingly, people did not rate the three robots differently in terms of competence or discomfort. Open answers revealed that the robot's resemblance with for example, a pet or a toy, and its positive social traits may be a reason to prefer the wooden or furry robot, whereas the plastic robot may be preferred because of its perceived usefulness.

As people have shown the same tendency to form impressions for robots as they do for humans [21], our findings may be explained by what we know about human impression formation. From social psychology, it is known that warmth and competence are the primary focus in impression formation among people. Specifically, wood as a furnishing material has been shown to be perceived as a warmer, more natural, and more inviting material, compared to glass or plastic [28]. Fur may remind people of (stuffed) animals, making the robot appear more organic and causing people to imbue it with emotion, as has been seen in animal-like robots [23], even when the robot is minimalistic in design [3]. 
The robots in this study were small, not highly detailed, table-top robots, which may explain why participants did not rate them differently on the discomfort dimension, as they were not uncanny [22]. In contrast to warmth, which reflects characteristics that benefit others, for example, whether someone is friendly, competence reflects characteristics that benefit the self, for example, being intelligent or skilled [12]. Competence is related to people's expectations of the robot's function, e.g., which task or what behavior they expect the robot to perform, but the robots did not have a specific task or function, which may explain why the robots were note rated differently in terms of competence.

Another interpretation for why we found an effect for warmth but not competence is that morality judgments may be more important in a first evaluation of someone. Fiske et al. [8, p. 77] argued that "[f]rom an evolutionary perspective, the primacy of warmth is fitting because another person's intent for good or ill is more important to survival than whether the other person can act on those intentions." Similarly, people's ratings of warmth may carry more weight than competence ratings when forming their first impressions of robots based on their outer appearance, where the weighting may have been further impacted by unspecified function of the three robots.

However, effects in video have been shown to differ between live results [2], and to develop real-world human-robot interactions, we need to validate our findings with live results. Also, the robots in this study did not have a function, which may have made it difficult to rate their perceived competence. Prior work showed that robot appearance affects which roles people see the robot fit [20]. As robots will take on specific roles in our daily lives, it is important to further investigate the effects of robot appearance on ratings of competence and discomfort when the robots are assigned specific tasks. Third, the sample of participants included both a large portion of people who worked with or studied robots and a large portion of people who did not interact with robots before. Hence, future work needs to determine whether there is a difference between the first impressions of people with different levels of experience with robots.

To summarize, we studied how component material (wood, synthetic fur, and plastic) affects people's first impressions of social robots. We found that people rated the plastic robot lower on warmth than the wooden and fur robot. Open answers revealed that people felt familiar with the wooden and fur robot, as they reminded them of pets and toys. We did not find differences in ratings of competence, discomfort, which may be explained by the fact that the robots lacked a specific function and by their benign appearance. Although the robots were equally preferred, the reasons behind this seem to be influenced by their component material, which needs to be further studied in future research.

\section{Acknowledgments}

This work was partially funded by a grant from the Swedish Research Council (reg. number 2017-05189). 


\section{References}

1. Bernotat, J., Eyssel, F., Sachse, J.: Shape it-the influence of robot body shape on gender perception in robots. In: International Conference on Social Robotics. pp. 75-84. Springer (2017)

2. Bucci, P., Cang, X.L., Valair, A., Marino, D., Tseng, L., Jung, M., Rantala, J., Schneider, O.S., MacLean, K.E.: Sketching cuddlebits: coupled prototyping of body and behaviour for an affective robot pet. In: Proceedings of the 2017 CHI Conference on Human Factors in Computing Systems. pp. 3681-3692. ACM (2017)

3. Bucci, P., Zhang, L., Cang, X.L., MacLean, K.E.: Is it happy?: Behavioural and narrative frame complexity impact perceptions of a simple furry robot's emotions. In: Proceedings of the 2018 CHI Conference on Human Factors in Computing Systems. p. 509. ACM (2018)

4. Carpinella, C.M., Wyman, A.B., Perez, M.A., Stroessner, S.J.: The robotic social attributes scale (rosas): Development and validation. In: Proceedings of the 2017 ACM/IEEE International Conference on human-robot interaction. pp. 254-262. $\operatorname{ACM}(2017)$

5. Crippa, G., Rognoli, V., Levi, M.: Materials and emotions, a study on the relations between materials and emotions in industrial products. In: 8 international conference on Design \& Emotion: out of control. pp. 1-9. Central Saint Martins College of Arts \& Design, London, England. (2012)

6. DiSalvo, C.F., Gemperle, F., Forlizzi, J., Kiesler, S.: All robots are not created equal: the design and perception of humanoid robot heads. In: Proceedings of the 4th conference on Designing interactive systems: processes, practices, methods, and techniques. pp. 321-326. ACM (2002)

7. Eyssel, F., De Ruiter, L., Kuchenbrandt, D., Bobinger, S., Hegel, F.: if you sound like me, you must be more human: On the interplay of robot and user features on human-robot acceptance and anthropomorphism. In: 2012 7th ACM/IEEE International Conference on Human-Robot Interaction (HRI). pp. 125-126. IEEE (2012)

8. Fiske, S.T., Cuddy, A.J., Glick, P.: Universal dimensions of social cognition: Warmth and competence. Trends in cognitive sciences 11(2), 77-83 (2007)

9. Green, R.D., MacDorman, K.F., Ho, C.C., Vasudevan, S.: Sensitivity to the proportions of faces that vary in human likeness. Computers in Human Behavior 24(5), 2456-2474 (2008)

10. Hegel, F., Eyssel, F., Wrede, B.: The social robot flobi: key concepts of industrial design. In: 19th International Symposium in Robot and Human Interactive Communication. pp. 107-112. IEEE (2010)

11. Hoffmann, L., Krämer, N.C.: Investigating the effects of physical and virtual embodiment in task-oriented and conversational contexts. International Journal of Human-Computer Studies 71(7-8), 763-774 (2013)

12. Holoien, D.S., Fiske, S.T.: Downplaying positive impressions: Compensation between warmth and competence in impression management. Journal of Experimental Social Psychology 49(1), 33-41 (2013)

13. Hu, Y., Hoffman, G.: Using skin texture change to design emotion expression in social robots. In: 2019 14th ACM/IEEE International Conference on Human-Robot Interaction (HRI). pp. 2-10. IEEE (2019)

14. Kalegina, A., Schroeder, G., Allchin, A., Berlin, K., Cakmak, M.: Characterizing the design space of rendered robot faces. In: Proceedings of the 2018 ACM/IEEE International Conference on Human-Robot Interaction. pp. 96-104. ACM (2018) 
15. Kennedy, J., Baxter, P., Belpaeme, T.: Comparing robot embodiments in a guided discovery learning interaction with children. International Journal of Social Robotics 7(2), 293-308 (2015)

16. Kirilenko, A.P., Stepchenkova, S.: Inter-coder agreement in one-to-many classification: fuzzy kappa. PloS one 11(3), e0149787 (2016)

17. Krach, S., Hegel, F., Wrede, B., Sagerer, G., Binkofski, F., Kircher, T.: Can machines think? interaction and perspective taking with robots investigated via fmri. PloS one 3(7), e2597 (2008)

18. Lehmann, H., Sureshbabu, A.V., Parmiggiani, A., Metta, G.: Head and face design for a new humanoid service robot. In: International Conference on Social Robotics. pp. 382-391. Springer (2016)

19. Lucas, H., Poston, J., Yocum, N., Carlson, Z., Feil-Seifer, D.: Too big to be mistreated? examining the role of robot size on perceptions of mistreatment. In: 2016 25th IEEE International Symposium on Robot and Human Interactive Communication (RO-MAN). pp. 1071-1076. IEEE (2016)

20. Luria, M., Forlizzi, J., Hodgins, J.: The effects of eye design on the perception of social robots. In: 2018 27th IEEE International Symposium on Robot and Human Interactive Communication (RO-MAN). pp. 1032-1037. IEEE (2018)

21. Mieczkowski, H., Liu, S.X., Hancock, J., Reeves, B.: Helping not hurting: Applying the stereotype content model and bias map to social robotics. In: 2019 14th ACM/IEEE International Conference on Human-Robot Interaction (HRI). pp. 222-229. IEEE (2019)

22. Mori, M., MacDorman, K.F., Kageki, N.: The uncanny valley [from the field]. IEEE Robotics \& Automation Magazine 19(2), 98-100 (2012)

23. Nakata, T., Sato, T., Mori, T., Mizoguchi, H.: Expression of emotion and intention by robot body movement. In: Proceedings of the 5th international conference on autonomous systems (1998)

24. Peña, D., Tanaka, F.: Validation of the design of a robot to study the thermoemotional expression. In: International Conference on Social Robotics. pp. 75-85. Springer (2018)

25. Phillips, E., Zhao, X., Ullman, D., Malle, B.F.: What is human-like?: Decomposing robots' human-like appearance using the anthropomorphic robot (abot) database. In: Proceedings of the 2018 ACM/IEEE International Conference on Human-Robot Interaction. pp. 105-113. ACM (2018)

26. Powers, A., Kiesler, S.: The advisor robot: tracing people's mental model from a robot's physical attributes. In: Proceedings of the 1st ACM SIGCHI/SIGART conference on Human-robot interaction. pp. 218-225. ACM (2006)

27. Premack, D., Woodruff, G.: Does the chimpanzee have a theory of mind? Behavioral and brain sciences 1(4), 515-526 (1978)

28. Rice, J., Kozak, R.A., Meitner, M.J., Cohen, D.H.: Appearance wood products and psychological well-being. Wood and Fiber Science 38(4), 644-659 (2007)

29. Short, Y.F.E., Short, D., Mataric, M.J.: Sprite: Stewart platform robot for interactive tabletop engagement. Department of Computer Science, University of Southern California, Tech Report (2017)

30. Wainer, J., Feil-Seifer, D.J., Shell, D.A., Mataric, M.J.: The role of physical embodiment in human-robot interaction. In: ROMAN 2006-The 15th IEEE International Symposium on Robot and Human Interactive Communication. pp. 117-122. IEEE (2006)

31. Willis, J., Todorov, A.: First impressions: Making up your mind after a 100-ms exposure to a face. Psychological science 17(7), 592-598 (2006) 